\title{
Fast Converging Semi-Blind Space-Time Equalisation for Dispersive QAM MIMO Systems
}

\author{
S. Chen and L. Hanzo
}

\begin{abstract}
A novel semi-blind space-time equaliser (STE) is proposed for dispersive multiple-input multiple-output systems that employ high-throughput quadrature amplitude modulation signalling. A minimum number of training symbols, approximately equal to the dimension of the STE, are first utilised to provide a rough initial least squares estimate of the STE's weight vector. A concurrent gradient-Newton constant modulus algorithm and soft decision-directed scheme is then applied to adapt the STE. The proposed semi-blind adaptive STE is capable of converging fast to the minimum mean square error STE solution. Simulation results confirms that the convergence speed of this semi-blind adaptive algorithm is very close to that of the training-based recursive least squares algorithm.
\end{abstract}

Index Terms-Multiple-input multiple-output, quadrature amplitude modulation, space-time equalisation, stochastic-gradient adaptive algorithm, gradient-Newton adaptive algorithm.

\section{INTRODUCTION}

W ITH the aid of smart antenna arrays and by exploiting both the space and time dimensions, space-time processing is capable of effectively improving the achievable system capacity, coverage and quality of service by suppressing both intersymbol interference and co-channel interference [1]-[7]. For the sake of further improving the achievable bandwidth efficiency, high-throughput quadrature amplitude modulation (QAM) schemes [8] have become popular in numerous wireless network standards. For example, the 16QAM and 64-QAM schemes were adopted in the recent WiMax standard [9]. In this contribution, we consider spacedivision multiple-access (SDMA) induced frequency selective multiple-input multiple-output (MIMO) systems that employ high-order QAM signalling. A bank of space-time equalisers (STEs) [10]-[16] form the multiuser receiver. Adaptive implementation of STE can be realised using training based methods, such as the least mean square (LMS) or recursive least squares (RLS) algorithm [17]. However, a large number of training symbols is required to properly train a STE, which considerably reduces the achievable system throughput.

Potentially, blind adaptive methods can be applied to adjust a STE, which does not require training symbols and, therefore, does not reduce the achievable system throughput. However, blind adaptive algorithms typically require high computational complexity and, moreover, blind methods result in unavoidable estimation and decision ambiguities [18], [19], which can only be resolved with the aid of a few training symbols. At the

Manuscript received January 8, 2009; revised March 16, 2009; accepted May 10, 2009. The associate editor coordinating the review of this letter and approving it for publication was R. Mallik.

The authors are with the School of Electronics and Computer Science, University of Southampton, Southampton SO17 1BJ, UK (e-mail: \{sqc, lh\}@ecs.soton.ac.uk).

Digital Object Identifier 10.1109/TWC.2009.090032 cost of requiring a few training symbols, semi-blind schemes can avoid the estimation and decision ambiguity problem and are computationally simpler than their blind counterparts. Many semi-blind methods [20]-[26] have been proposed for frequency nonselective MIMO systems. In particular, the work of [26] has developed a semi-blind spatial equalisation scheme for narrowband MIMO systems that employ high-order QAM signalling. In this semi-blind method, a few training symbols, approximately equal to the dimension $N_{\mathrm{SE}}$ of the spatial equaliser, are first used to provide a rough least squares (LS) estimate of the spatial equaliser's weight vector. The stochastic-gradient (SG) concurrent constant modulus algorithm (CMA) and soft decision-directed (SDD) scheme, originally developed for blind equalisation of single-input singleoutput dispersive systems [27], [28], is then employed to adapt the spatial equaliser. This semi-blind SG spatial equalisation scheme converges fast to the minimum mean square error (MMSE) solution with a computational complexity in the order of $N_{\mathrm{SE}}, \mathcal{O}\left(N_{\mathrm{SE}}\right)$, similar to the training-based LMS algorithm.

Direct adopting the semi-blind SG strategy for spatial equalisation of narrowband MIMO systems to adapt the STE that operates in frequency selective MIMO systems, however, is generally unwise. This is because, under dispersive MIMO environment, the STE's input signal is highly correlated and a SG-type adaptive algorithm suffers from slow convergence and high steady-state misadjustment [17]. The novelty of this contribution is that we propose a gradient-Newton (GN) based concurrent CMA and SDD algorithm to adapt the STE. GN-type algorithms [29] employ second-order statistics of input signal to "whiten" stochastic gradient, which results in much faster convergence than SG-type algorithms in highly correlated signal environments at the cost of an increased complexity. A GN-type algorithm has been adopted in the trainingbased adaptive multiuser receiver for dispersive MIMO systems [30]. Our proposed semi-blind GN-CMA+SDD adaptive algorithm is capable of converging fast to the optimal MMSE STE solution and it has a computational complexity in the order of $N_{\mathrm{STE}}^{2}, \mathcal{O}\left(N_{\mathrm{STE}}^{2}\right)$, similar to that of the trainingbased RLS algorithm, where $N_{\mathrm{STE}}$ denotes the dimension of the STE. Simulation study shows that the convergence speed of this semi-blind GN-CMA+SDD adaptive algorithm is very close to that of the RLS algorithm, while the semiblind SG-CMA+SDD adaptive algorithm converges too slow in the dispersive MIMO environment and is incapable of approaching the optimal MMSE STE solution due to the excessive steady-state misadjustment. 


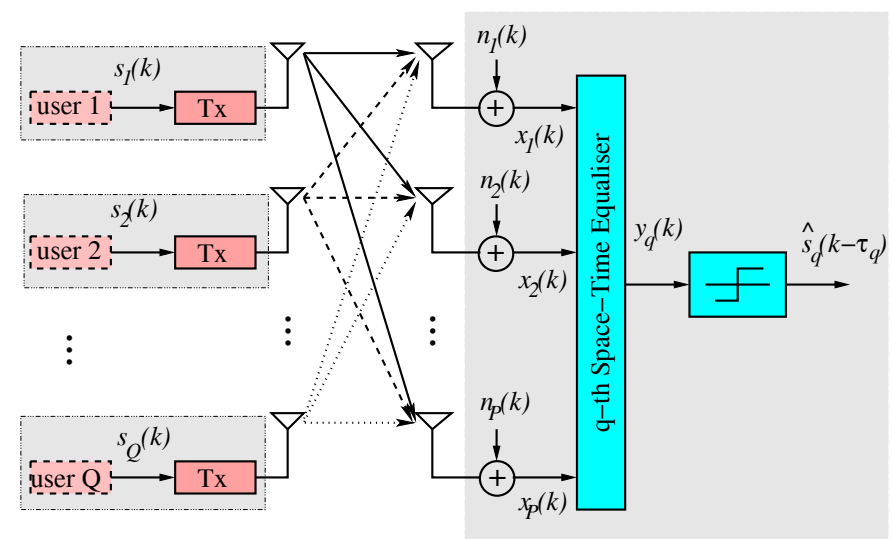

Fig. 1. SDMA induced MIMO system, where each of the $Q$ users is equipped with a single transmit antenna and the receiver is assisted by a $P$-element antenna array.

\section{System Model And STE Structure}

The SDMA induced MIMO system is depicted in Fig. 1, where each of the $Q$ users is equipped with a single transmit antenna and the receiver is assisted by a $P$-element antenna array. Denote the symbol-rate channel impulse response (CIR) connecting the $q$ th transmit antenna to the $p$ th receive antenna as $\mathbf{c}_{p, q}=\left[\begin{array}{lll}c_{0, p, q} & c_{1, p, q} \cdots c_{n_{C}-1, p, q}\end{array}\right]^{T}$, where for notational simplicity we have assumed that each of the $P \times Q$ CIRs has the same length of $n_{C}$. The symbol-rate received signal samples $x_{p}(k), 1 \leq p \leq P$, can be expressed as

$$
x_{p}(k)=\sum_{q=1}^{Q} \sum_{i=0}^{n_{C}-1} c_{i, p, q} s_{q}(k-i)+n_{p}(k),
$$

where $n_{p}(k)$ is a complex-valued Gaussian white noise process with $E\left[\left|n_{p}(k)\right|^{2}\right]=2 \sigma_{n}^{2}, s_{q}(k)$ is the $k$ th transmitted symbol of user $q$ with the symbol energy $E\left[\left|s_{q}(k)\right|^{2}\right]=\sigma_{s}^{2}$, and $s_{q}(k)$ takes the values from the $M$-QAM symbol set

$$
\mathcal{S} \triangleq\left\{s_{i, l}=u_{i}+j u_{l}, 1 \leq i, l \leq \sqrt{M}\right\}
$$

with the real-part symbol $\Re\left[s_{i, l}\right]=u_{i}=2 i-\sqrt{M}-1$ and the imaginary-part symbol $\Im\left[s_{i, l}\right]=u_{l}=2 l-\sqrt{M}-1$. The overall system's received signal-to-noise ratio (SNR) is defined as

$$
\mathrm{SNR}=\frac{\sum_{q=1}^{Q} \sum_{p=1}^{P} \mathbf{c}_{p, q}^{H} \mathbf{c}_{p, q} \sigma_{s}^{2}}{2 Q P \sigma_{n}^{2}} .
$$

The STE for detecting the $q$ th user's data is depicted in Fig. 2. The STE's output, given by

$$
y_{q}(k)=\sum_{p=1}^{P} \sum_{i=0}^{D-1} w_{i, p, q}^{*} x_{p}(k-i),
$$

is passed to the decision device to produce an estimate $\hat{s}_{q}(k-$ $\tau_{q}$ ) of the transmitted symbol $s_{q}\left(k-\tau_{q}\right)$, where $D$ is the temporal filter's length, $w_{i, p, q}$ are the weights of the STE, and $0 \leq \tau_{q} \leq D+n_{C}-2$ is the decision delay.

Define the overall received signal vector $\mathbf{x}(k)=$ $\left[\mathbf{x}_{1}^{T}(k) \mathbf{x}_{2}^{T}(k) \cdots \mathbf{x}_{P}^{T}(k)\right]^{T}$, where

$$
\mathbf{x}_{p}(k)=\left[x_{p}(k) x_{p}(k-1) \cdots x_{p}(k-D+1)\right]^{T},
$$

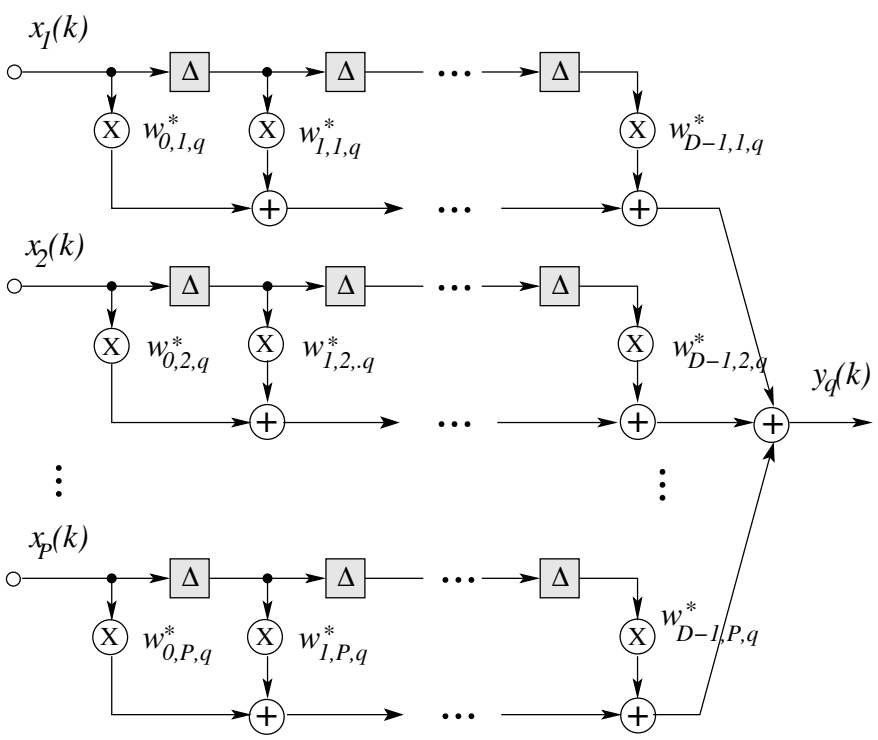

Fig. 2. Space-time equaliser for user $q$, where $\Delta$ denotes the symbol-spaced delay, $P$ is the number of receive antennas, $D$ denotes the length of temporal filter, and $1 \leq q \leq Q$ with $Q$ being the number of users.

for $1 \leq p \leq P$. Then $\mathbf{x}(k)$ can be expressed by the wellknown MIMO model

$$
\mathbf{x}(k)=\mathbf{C ~ s}(k)+\mathbf{n}(k)
$$

where $\mathbf{n}(k)=\left[\mathbf{n}_{1}^{T}(k) \mathbf{n}_{2}^{T}(k) \cdots \mathbf{n}_{P}^{T}(k)\right]^{T}$ with

$$
\mathbf{n}_{p}(k)=\left[n_{p}(k) n_{p}(k-1) \cdots n_{p}(k-D+1)\right]^{T}
$$

for $1 \leq p \leq P$, the tranmitted symbol vector of all the users $\mathbf{s}(k)=\left[\mathbf{s}_{1}^{T}(k) \mathbf{s}_{2}^{T}(k) \cdots \mathbf{s}_{Q}^{T}(k)\right]^{T}$ with

$$
\mathbf{s}_{q}(k)=\left[s_{q}(k) s_{q}(k-1) \cdots s_{q}\left(k-D-n_{C}+2\right)\right]^{T},
$$

for $1 \leq q \leq Q$, and the overall system's CIR matrix

$$
\mathbf{C}=\left[\begin{array}{cccc}
\mathbf{C}_{1,1} & \mathbf{C}_{1,2} & \cdots & \mathbf{C}_{1, Q} \\
\mathbf{C}_{2,1} & \mathbf{C}_{2,2} & \cdots & \mathbf{C}_{2, Q} \\
\vdots & \vdots & \cdots & \vdots \\
\mathbf{C}_{P, 1} & \mathbf{C}_{P, 2} & \cdots & \mathbf{C}_{P, Q}
\end{array}\right]
$$

with the $D \times\left(D+n_{C}-1\right)$ CIR matrix associated with the user $q$ and the receive antenna $p$ given by the Toeplitz form

$$
\mathbf{C}_{p, q}=\left[\begin{array}{cccc}
\mathbf{c}_{p, q}^{T} & 0 & \cdots & 0 \\
0 & \mathbf{c}_{p, q}^{T} & \ddots & \vdots \\
\vdots & \ddots & \ddots & 0 \\
0 & \cdots & 0 & \mathbf{c}_{p, q}^{T}
\end{array}\right]
$$

for $1 \leq p \leq P$ and $1 \leq q \leq Q$.

Similarly, the STE for detecting the $q$ th user's data can be expressed in the vector form

$$
y_{q}(k)=\mathbf{w}_{q}^{H} \mathbf{x}(k)
$$

where the overall weight vector of the STE $\mathbf{w}_{q}=$ $\left[\mathbf{w}_{1, q}^{T} \mathbf{w}_{2, q}^{T} \cdots \mathbf{w}_{P, q}^{T}\right]^{T}$ with

$$
\mathbf{w}_{p, q}=\left[w_{0, p, q} w_{1, p, q} \cdots w_{D-1, p, q}\right]^{T}, 1 \leq p \leq P .
$$


The dimension of the STE is thereofore $N_{\mathrm{STE}}=P$. $D$. The mean square error (MSE) value $J_{\mathrm{MSE}}\left(\mathbf{w}_{q}\right)=$ $E\left[\left|s_{q}\left(k-\tau_{q}\right)-y_{q}(k)\right|^{2}\right]$ of the STE (11) is given by

$$
\begin{aligned}
J_{\mathrm{MSE}}\left(\mathbf{w}_{q}\right) & =\sigma_{s}^{2}\left(1-\mathbf{w}_{q}^{H} \mathbf{C}_{\mid q_{\eta}}-\mathbf{w}_{q}^{T} \mathbf{C}_{\mid q_{\eta}}^{*}\right) \\
& +\sigma_{s}^{2} \mathbf{w}_{q}^{H}\left(\mathbf{C} \mathbf{C}^{H}+\frac{2 \sigma_{n}^{2}}{\sigma_{s}^{2}} \mathbf{I}\right) \mathbf{w}_{q},
\end{aligned}
$$

where I denotes the $N_{\mathrm{STE}} \times\left(Q \cdot\left(D+n_{C}-1\right)\right)$ dimensional identity matrix, $q_{\eta}=(q-1)\left(D+n_{C}-1\right)+\left(\tau_{q}+1\right)$ and $\mathbf{C}_{\mid i}$ the $i$ th column of $\mathbf{C}$. Define the impulse response of the combined STE (11) and MIMO channel as

$$
\mathbf{f}_{q}^{T}=\left[f_{0, q} f_{1, q} \cdots f_{\tau_{\max }, q}\right]=\mathbf{w}_{q}^{H} \mathbf{C}
$$

where $\tau_{\max }=\left(D+n_{C}-1\right) \cdot Q-1$, and let

$$
i_{\max , q}=\arg \max _{0 \leq i \leq \tau_{\max }}\left|f_{i, q}\right|,
$$

where, in fact, $i_{\max , q}=q_{\eta}$. In the simulation, the quality of equalisation can alternatively be assessed using the maximum distortion (MD) measure defined by

$$
J_{\mathrm{MD}}\left(\mathbf{w}_{q}\right)=\left(\sum_{i=0}^{\tau_{\max }}\left|f_{i, q}\right|-\left|f_{i_{\max }, q}\right|\right) /\left|f_{i_{\max }, q}\right| .
$$

Ultimately, the symbol error rate (SER) can be simulated to assess the equalisation performance. With the perfect channel knowledge, the optimal MMSE solution for the STE (11) is

$$
\mathbf{w}_{q(\mathrm{MMSE})}=\left(\mathbf{C C}^{H}+\frac{2 \sigma_{n}^{2}}{\sigma_{s}^{2}} \mathbf{I}\right)^{-1} \mathbf{C}_{\mid q_{\eta}} .
$$

Owing to space limitation, in the simulation study, instead of showing the individual STE's performance, we opt for displaying the average SER over all the $Q$ users, the average MSE

$$
J_{\mathrm{AMSE}}(\mathbf{W})=\frac{1}{Q} \sum_{q=1}^{Q} J_{\mathrm{MSE}}\left(\mathbf{w}_{q}\right),
$$

and the average $\mathrm{MD}$

$$
J_{\mathrm{AMD}}(\mathbf{W})=\frac{1}{Q} \sum_{q=1}^{Q} J_{\mathrm{MD}}\left(\mathbf{w}_{q}\right),
$$

where $\mathbf{W}=\left[\mathbf{w}_{1} \mathbf{w}_{2} \cdots \mathbf{w}_{Q}\right]$ denotes the weight matrix of all the $Q$ STEs.

\section{The Proposed Semi-Blind Algorithm}

Let the number of available training symbols be $K$, and denote the available training data as

$$
\left\{\begin{array}{l}
\mathbf{X}_{K}=[\mathbf{x}(1) \mathbf{x}(2) \cdots \mathbf{x}(K)], \\
\overline{\mathbf{s}}_{K, q}=\left[s_{q}\left(1-\tau_{q}\right) s_{q}\left(2-\tau_{q}\right) \cdots s_{q}\left(K-\tau_{q}\right)\right]^{T} .
\end{array}\right.
$$

The LS estimate of the STE's weight vector based on $\left\{\mathbf{X}_{K}, \overline{\mathbf{s}}_{K, q}\right\}$ is readily given as

$$
\mathbf{w}_{q}(0)=\left(\mathbf{X}_{K} \mathbf{X}_{K}^{H}\right)^{-1} \mathbf{X}_{K} \overline{\mathbf{s}}_{K, q}^{*} .
$$

In order to maintain throughput, the number of training pilots should be as small as possible. To ensure that $\mathbf{X}_{K} \mathbf{X}_{K}^{H}$ has a full rank, we will choose $K$ slightly larger than $N_{\mathrm{STE}}$, the dimension of $\mathbf{x}(k)$. Because the training data with $K \approx N_{\mathrm{STE}}$ are generally insufficient, the initial LS weight vector (21) may not be sufficiently accurate to open the eye. Therefore, decision direct adaptation is generally unsafe. Also directly apply the SG-CMA+SDD blind scheme of [27] to adapt the STE (11) with $\mathbf{w}_{q}(0)$ of (21) as the initial weight vector suffers from slow convergence and high steady-state MSE misadjustment, because $\mathbf{x}(k)$ is highly correlated. We propose a GN-CMA+SDD algorithm for adjusting the STE (11) with $\mathbf{w}_{q}(0)$ of (21) as the initial weight vector, which is capable of converging fast and accurately to the MMSE STE solution.

A GN algorithm [29] uses the inverse of the autocorrelation matrix of $\mathbf{x}(k)$ to modify the stochastic gradient. Just like in the RLS algorithm, this inverse matrix can be updated recursively according to [17]

$$
\mathbf{P}(k)=\lambda^{-1} \mathbf{P}(k-1)-\lambda^{-1} \mathbf{g}(k) \mathbf{x}^{H}(k) \mathbf{P}(k-1)
$$

with

$$
\mathbf{g}(k)=\frac{\lambda^{-1} \mathbf{P}(k-1) \mathbf{x}(k)}{1+\lambda^{-1} \mathbf{x}^{H}(k) \mathbf{P}(k-1) \mathbf{x}(k)},
$$

where $\lambda \leq 1$ is the forgetting factor [17]. For stationary channels, $\lambda=1$ is appropriate. The initial $\mathbf{P}(0)$ can be set to $\mathbf{P}(0)=\left(\mathbf{X}_{K} \mathbf{X}_{K}^{H}\right)^{-1}$. Let the STE's weight vector be split into two parts, yielding $\mathbf{w}_{q}=\mathbf{w}_{q, c}+\mathbf{w}_{q, d}$. The initial $\mathbf{w}_{q, c}$ and $\mathbf{w}_{q, d}$ are simply set to $\mathbf{w}_{q, c}(0)=\mathbf{w}_{q, d}(0)=0.5 \mathbf{w}_{q}(0)$. Denote the STE's output at sample $k$ as $y_{q}(k)=\mathbf{w}_{q}^{H}(k) \mathbf{x}(k)$.

The weight vector $\mathbf{w}_{q, c}$ is updated using the GN-CMA according to

$$
\mathbf{w}_{q, c}(k+1)=\mathbf{w}_{q, c}(k)+\mu_{\mathrm{CMA}} \mathbf{P}(k) \varepsilon^{*}(k) \mathbf{x}(k)
$$

with

$$
\varepsilon(k)=y_{q}(k)\left(\Delta-\left|y_{q}(k)\right|^{2}\right),
$$

where $\Delta=E\left[\left|s_{q}(k)\right|^{4}\right] / E\left[\left|s_{q}(k)\right|^{2}\right]$ and $\mu_{\mathrm{CMA}}$ is the step size of the CMA. It is obvious that this GN-CMA algorithm reduces to the conventional SG-CMA [31], [32] if $\mathbf{P}(k)$ is replaced with an identity matrix. It is well-known that the step size for the SG-CMA must be chosen sufficiently small to avoid divergence, particularly in a highly correlated signal environment. By contrast, the step size of the GN-CMA algorithm can be set to a value much larger than the step size of the SG-CMA counterpart.

The weight vector $\mathbf{w}_{q, d}$ is updated using the GN-SDD scheme, which is now described. The complex phasor plane is divided into the $M / 4$ rectangular regions, and each region $\mathcal{S}_{i, l}$ contains four symbol points as defined in the following

$$
\mathcal{S}_{i, l}=\left\{s_{r, m}, \quad r=2 i-1,2 i, m=2 l-1,2 l\right\},
$$

where $1 \leq i, l \leq \sqrt{M} / 2$. If the STE's output $y_{q}(k) \in \mathcal{S}_{i, l}$, a local approximation of the marginal probability density function (PDF) of $y_{q}(k)$ is given by [27], [28]

$$
\hat{p}\left(\mathbf{w}_{q}, y_{q}(k)\right) \approx \sum_{r=2 i-1}^{2 i} \sum_{m=2 l-1}^{2 l} \frac{1}{8 \pi \rho} e^{-\frac{\left|y_{q}(k)-s_{r, m}\right|^{2}}{2 \rho}},
$$

where $\rho$ is the cluster width associated with the four clusters of each $\mathcal{S}_{i, l}$. The SG-SDD algorithm [27], [28] is designed to maximise the $\log$ of the local marginal PDF criterion $E\left[J_{\mathrm{LMAP}}\left(\mathbf{w}_{q}, k\right)\right]$, where $J_{\mathrm{LMAP}}\left(\mathbf{w}_{q}, k\right)=$ $\rho \log \left(\hat{p}\left(\mathbf{w}_{q}, y_{q}(k)\right)\right)$, via a stochastic gradient optimisation. 
TABLE I

CIRS FOR THE 3-USER 4-ANTENNA 16-QAM MIMO SYSTEM.

\begin{tabular}{c|c|c|c}
\hline $\mathbf{c}_{p, q}$ & $q=1$ & $q=2$ & $q=3$ \\
\hline$p=1$ & $-0.424+j 0.339$ & $-0.095-j 0.191$ & $-0.516+j 0.664$ \\
& $+0.594+j 0.509$ & $+0.667+j 0.572$ & $+0.442+j 0.295$ \\
& $+0.255-j 0.170$ & $+0.381+j 0.191$ & $-0.074+j 0.074$ \\
\hline$p=2$ & $+0.432-j 0.346$ & $-0.223+j 0.372$ & $-0.419+j 0.559$ \\
& $-0.691-j 0.259$ & $-0.520-j 0.669$ & $-0.419-j 0.489$ \\
& $+0.173+j 0.346$ & $+0.074+j 0.297$ & $-0.279-j 0.140$ \\
\hline$p=3$ & $+0.306-j 0.306$ & $-0.093-j 0.186$ & $+0.253-j 0.421$ \\
& $-0.535-j 0.612$ & $+0.650+j 0.557$ & $+0.758+j 0.084$ \\
& $+0.382+j 0.077$ & $+0.464+j 0.093$ & $+0.337-j 0.253$ \\
\hline$p=4$ & $+0.385+j 0.385$ & $-0.479-j 0.319$ & $-0.505-j 0.505$ \\
& $+0.462-j 0.692$ & $+0.718-j 0.319$ & $+0.674+j 0.000$ \\
& $-0.077-j 0.077$ & $+0.160+j 0.160$ & $+0.168+j 0.084$ \\
\hline
\end{tabular}

By contrast, the proposed GN-SDD algorithm uses $\mathbf{P}(k)$ to modify the stochastic gradient and updates $\mathbf{w}_{q, d}$ according to

$\mathbf{w}_{q, d}(k+1)=\mathbf{w}_{q, d}(k)+\mu_{\mathrm{SDD}} \mathbf{P}(k) \frac{\partial J_{\mathrm{LMAP}}\left(\mathbf{w}_{q}(k), k\right)}{\partial \mathbf{w}_{q, d}}$,

where $\mu_{\mathrm{SDD}}$ is the step size of the SDD, and

$$
\begin{aligned}
\frac{\partial J_{\mathrm{LMAP}}\left(\mathbf{w}_{q}, k\right)}{\partial \mathbf{w}_{q, d}}= & \frac{1}{Z_{N}} \sum_{r=2 i-1}^{2 i} \sum_{m=2 l-1}^{2 l} e^{-\frac{\left|y_{q}(k)-s_{r, m}\right|^{2}}{2 \rho}} \\
& \times\left(s_{r, m}-y_{q}(k)\right)^{*} \mathbf{x}(k)
\end{aligned}
$$

with

$$
Z_{N}=\sum_{r=2 i-1}^{2 i} \sum_{m=2 l-1}^{2 l} e^{-\frac{\left|y_{q}(k)-s_{r, m}\right|^{2}}{2 \rho}} .
$$

This GN-SDD algorithm reduces to the SG-SDD algorithm of [27], [28] by replacing $\mathbf{P}(k)$ with an identity matrix. Note that, for the SG-SDD algorithm, the step size $\mu_{\mathrm{SDD}}$ has significant influence on the performance of the algorithm. Too large value of $\mu_{\text {SDD }}$ results in divergence while too small value of $\mu_{\text {SDD }}$ leads to slow convergence. By contrast, there is no need in choosing $\mu_{\mathrm{SDD}}$ for the GN-SDD algorithm, as it can simply be set to 1.0 or a value smaller than but close to 1.0 , just as in the training-based RLS algorithm. The performance of the GN-SDD algorithm is insensitive to the cluster width $\rho$, defined in the context of the local PDF (27), just as in the case of the SG-SDD. Detailed discussion on the influence of $\rho$ to the performance of the algorithm can be found in [26]-[28].

It is also clear that the proposed GN-CMA+SDD algorithm has a complexity in the order of $N_{\mathrm{STE}}^{2}, \mathcal{O}\left(N_{\mathrm{STE}}^{2}\right)$, similar to that of the RLS algorithm, while the SG-CMA+SDD algorithm has a complexity in the order of $N_{\mathrm{STE}}, \mathcal{O}\left(N_{\mathrm{STE}}\right)$, similar to that of the LMS algorithm.

\section{Simulation Study}

The system used in our simulation supported $Q=3$ users with $P=4$ receive antennas, and the modulation scheme was 16-QAM. The $P \cdot Q=12$ CIRs $\mathbf{c}_{p, q}, 1 \leq p \leq 4$ and $1 \leq q \leq 3$, are listed in Table I, each CIR having $n_{C}=3$ taps. The STE's temporal filter order was chosen as $D=5$. The optimal decision delays were found to be $\tau_{1}=4$ for user one, $\tau_{2}=3$ for user two and $\tau_{3}=2$ for user three. These decision delays were used in our simulation. Note that there was a trade off in choosing an appropriate temporal filter length $D$. A larger $D$ offered better capability of removing intersymbol

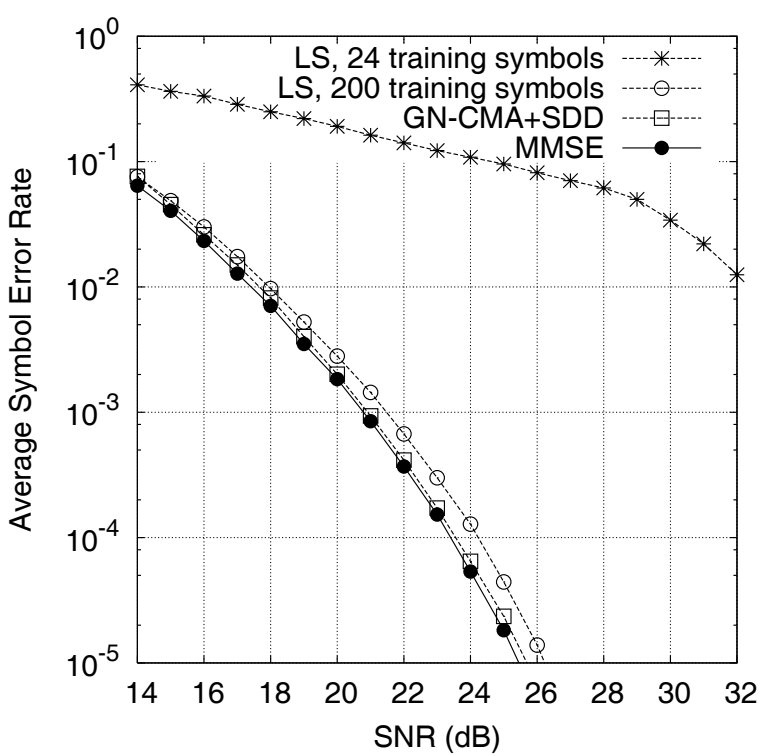

Fig. 3. Comparsion of the average symbol error rate performance for the training-based LS, semi-blind GN-CMA+SDD, and optimal MMSE STEs.

interference but resulted in longer adaptation period and higher steady-state misadjustment, which was a particular problem for SG-type adaptive algorithm. The average SER over all the $Q=3$ optimal MMSE STEs, depicted in Fig. 3, was used as the benchmark performance. The LS training-based STEs were also tested. Given the training data $\left\{\mathbf{X}_{K}, \overline{\mathbf{s}}_{K, q}\right\}$, the LS estimate of the STE weight vector was provided by (21), and the average SER performance of the three LS training-based STEs were also depicted in Figs. 3, given $K=24$ and $K=$ 200 , respectively. It can be seen that $K=24$ was insufficient for the LS training based STEs to achieve an adequate SER performance and at least $K=200$ training symbols were required by the STEs to approximate the optimal MMSE STE solutions.

The proposed semi-blind STE was next investigated. Given a SNR value, $K=24$ training pilots were first used to provide the initial weight vector of the STE according to (21). The GN-CMA+SDD blind algorithm then adapted the STE. The convergence performance of the proposed GNCMA+SDD algorithm was investigated, in comparison with the SG-CMA+SDD algorithm of [26]. For all the three blind SG-CMA+SDD STEs, $\mu_{\mathrm{CMA}}=0.00001, \mu_{\mathrm{SDD}}=0.0002$ and $\rho=0.1$ were chosen, while $\mu_{\mathrm{CMA}}=0.01, \mu_{\mathrm{SDD}}=0.95$ and $\rho=0.1$ were used for all the three blind GN-CMA+SDD STEs. These parameters were found empirically to yield the best performance in terms of convergence speed and steadystate misadjustment. Note that the step size values of the GNCMA+SDD based semi-blind STEs were much larger than their counterparts for the SG-CMA+SDD based semi-blind STEs, and the GN-CMA+SDD adaptive algorithm was also seen to be insensitive to the value of $\rho$. Figs. 4 and 5 plot the learning curves of the GN-CMA+SDD adaptive algorithm for the three users obtained by averaging over 50 different runs, in terms of the average MSE $J_{\mathrm{AMSE}}(\mathbf{W}(k))$ and the average MD meaure $J_{\mathrm{AMD}}(\mathbf{W}(k))$, respectively, in comparison with those obtained by the SG-CMA+SDD based STEs as well as the results obtained by the training-based RLS STEs. As expected, under a highly dispersive MIMO environment, the 


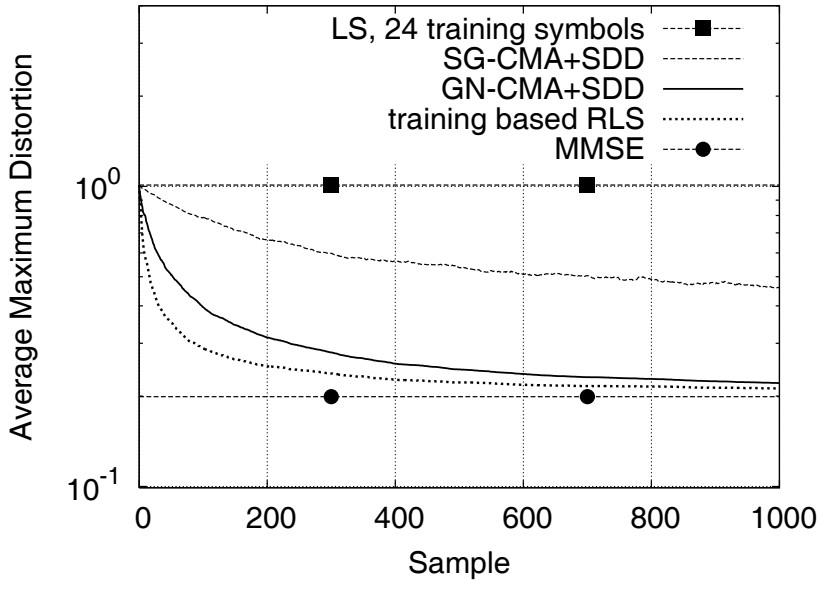

Fig. 4. Convergence performance of the SG-CMA+SDD, GN-CMA+SDD and training-based RLS STEs in terms of the average maximum distortion, given SNR of $21 \mathrm{~dB}$ and averaged over 50 runs.

SG-CMA+SDD algorithm converged very slowly and was incapable of approaching the optimal MMSE STE solution due to a excessively high steady-state misadjustment. By contrast, the proposed GN-CMA+SDD algorithm was capable of converging fast and accurately to the optimal MMSE STE solution. Next, given a range of SNR values, the average SER performance of the three GN-CMA+SDD based semi-blind STEs after adaptation of 1000 samples, were plotted in Fig. 3, in comparion with those of the optimal MMSE STEs and the LS training-based STEs.

\section{Conclusions}

A semi-blind STE scheme has been proposed for frequency selective MIMO systems that employ high throughput QAM signalling. A minimum number of training symbols, approximately equal to the dimension of the STE, is used to provide a rough LS estimate of the STE weight vector for the initialisation. A novel GN-CMA+SDD blind adaptive scheme is then adopted to adapt the STE. The proposed semi-blind STE scheme has a complexity similar to that of the RLS algorithm, and it is capable of converging fast and accurately to the optimal MMSE STE solution calculated based on the perfect channel knowledge. Our simulation study has confirmed that this semi-blind GN-CMA+SDD algorithm has a convergence speed very close to the training-based RLS algorithm under the highly dispersive MIMO environment.

\section{REFERENCES}

[1] G. J. Foschini, "Layered space-time architecture for wireless communication in a fading environment when using multiple antennas," Bell Labs Tech. J., vol. 1, pp. 41-59, 1996.

[2] J. H. Winters, "Smart antennas for wireless systems," IEEE Personal Commun., vol. 5, pp. 23-27, Feb. 1998.

[3] P. Vandenameele, L. van Der Perre, and M. Engels, Space Division Multiple Access for Wireless Local Area Networks. Boston, MA: Kluwer Academic Publishers, 2001.

[4] J. S. Blogh and L. Hanzo, Third Generation Systems and Intelligent Wireless Networking - Smart Antennas and Adaptive Modulation. Chichester, UK: Wiley, 2002.

[5] A. Paulraj, R. Nabar and D. Gore, Introduction to Space-Time Wireless Communications. Cambridge, UK: Cambridge University Press, 2003.

[6] A. J. Paulraj, D. A. Gore, R. U. Nabar, and H. Bölcskei, "An overview of MIMO communications - a key to gigabit wireless," Proc. IEEE, vol. 92, pp. 198-218, Feb. 2004.

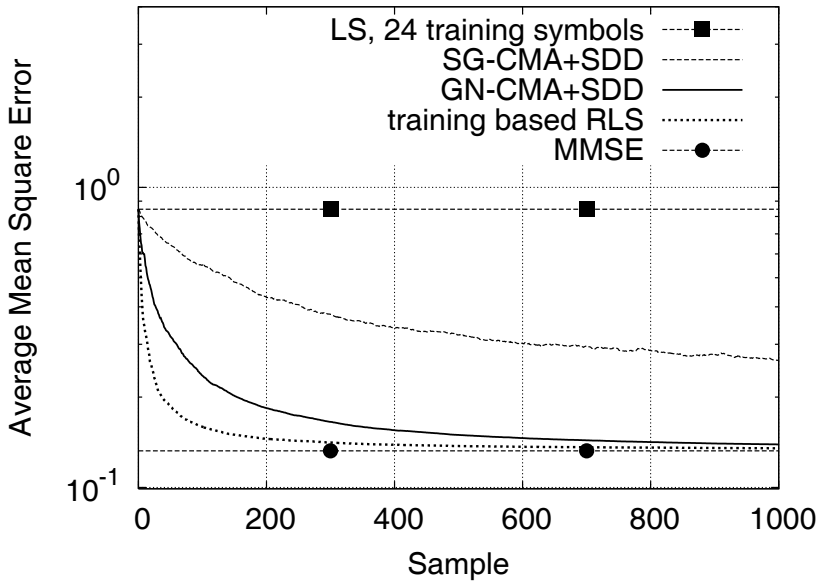

Fig. 5. Convergence performance of the SG-CMA+SDD, GN-CMA+SDD and training-based RLS STEs in terms of the average mean square error, given SNR of $21 \mathrm{~dB}$ and averaged over 50 runs.

[7] D. Tse, and P. Viswanath, Fundamentals of Wireless Communication. Cambridge, UK: Cambridge University Press, 2005.

[8] L. Hanzo, S. X. Ng, T. Keller and W. Webb, Quadrature Amplitude Modulation: From Basics to Adaptive Trellis-Coded, Turbo-Equalised and Space-Time Coded OFDM, CDMA and MC-CDMA Systems. Chichester, UK: John Wiley and IEEE Press, 2004.

[9] IEEE 802.16, Air Interface for Fixed Broadband Wireless Access System, Section 8. PHY, 2004.

[10] U. Trautwein, G. Sommerkorn, and R. S. Thomä, "A simulation study on space-time equalization for mobile broadband communication in an industrial indoor environment," in Proc. VTC 1999-Spring, vol. 1, (Houston, TX), pp. 511-515, May 1999.

[11] U. Trautwein, D. Hampicke, G. Sommerkorn, and R. S. Thomä, "Performance of space-time processing for ISI- and CCI-suppression in industrial scenarios," in Proc. VTC 2000-Spring, vol. 3, Tokyo, Japan, pp. 1894-1898, May 2000.

[12] A. Lozano and C. Papadias, "Layered space-time receivers for frequency-selective wireless channels," IEEE Trans. Commun., vol. 50, pp. 65-73, Jan. 2002.

[13] X. Zhu and R. D. Murch, "Layered space-time equalization for wireless MIMO systems," IEEE Trans. Wireless Commun., vol. 2, pp. 1189-1203, Nov. 2003.

[14] S. Chen, A. Livingstone, and L. Hanzo, "Minimum bite-error rate design for space-time equalization-based multiuser detection," IEEE Trans. Commun., vol. 54, pp. 824-832, May 2006.

[15] S. Chen, L. Hanzo and A. Livingstone, "MBER Space-time decision feedback equalization assisted multiuser detection for multiple antenna aided SDMA systems," IEEE Trans. Signal Processing, vol. 54, pp. 3090-3098, Aug. 2006.

[16] Y. Lee and W.-R. Wu, "Adaptive decision feedback space-time equalization with generalized sidelobe cancellation," IEEE Trans. Veh. Technol., vol. 57, pp. 2894-2906, Sept. 2008.

[17] S. Haykin, Adaptive Filter Theory. Upper Saddle Rever, NJ: Prentice Hall, 3rd ed., 1996.

[18] L. Tang, R. W. Liu, V. C. Soon and Y. F. Huang, "Indeterminacy and identifiability of blind identification," IEEE Trans. Circuits and Systems, vol. 38, pp. 499-509, May 1991.

[19] Y. Inouye and R. W. Liu, "A system-theoretic foundation for blind equalization of an FIR MIMO channel system," IEEE Trans. Circuits and Systems Part I: Fundamental Theory and Applications, vol. 49, pp. 425-436, Apr. 2002.

[20] A. Medles and D. T. M. Slock, "Semiblind channel estimation for MIMO spatial multiplexing systems," in Proc. VTC2001-Fall, vol. 2, Atlantic City, NJ, pp. 1240-1244, Oct. 2001.

[21] C. Cozzo and B. L. Hughes, "Joint channel estimation and data detection in space-time communications," IEEE Trans. Commun., vol. 51, pp. 1266-1270, Aug. 2003.

[22] T. Wo, P. A. Hoeher, A. Scherb, and K. D. Kammeyer, "Performance analysis of maximum-likelihood semiblind estimation of MIMO channels," in Proc. VTC2006-Spring, vol. 4, Melbourne, Australia, pp. 17381742, May 2006.

[23] A. K. Jagannatham and B. D. Rao, "Whitening-rotation-based semi- 
blind MIMO channel estimation," IEEE Trans. Signal Processing, vol. 54, pp. 861-869, Mar. 2006.

[24] Z. Ding, T. Ratnarajah, and C. F. N. Cowan, "HOS-based semi-blind spatial equalization for MIMO Rayleigh fading channels," IEEE Trans. Signal Processing, vol. 56, pp. 248-255, Jan. 2008.

[25] M. Abuthinien, S. Chen and L. Hanzo, "Semi-blind joint maximum likelihood channel estimation and data detection for MIMO systems," IEEE Signal Processing Lett., vol. 15, pp. 202-205, 2008.

[26] S. Chen, W. Yao and L. Hanzo, "Semi-blind adaptive spatial equalisation for MIMO systems with high-order QAM signalling," IEEE Trans. Wireless Commun., vol. 7, pp. 4486-4491, Nov. 2008

[27] S. Chen and E. S. Chng, "Concurrent constant modulus algorithm and soft decision directed scheme for fractionally-spaced blind equalization," in Proc. ICC 2004, vol. 4, Paris, France, pp. 2342-2346, June 2004.

[28] S. Chen, T. B. Cook, and L. C. Anderson, "A comparative study of two blind FIR equalizers,", Digital Signal Processing, vol. 14, pp. 18-36, Jan. 2004.

[29] P. S. R. Diniz, Adaptive Filtering: Algorithms and Practical Implementation. Boston, MA: Kluwer Academic Publishers, 2nd ed., 2003.

[30] R. C. de Lamare and R. Sampaio-Neto, "Adaptive MBER decision feedback multiuser receivers in frequency selective fading channels," IEEE Commun. Lett., vol. 7, pp. 73-75, Feb. 2003.

[31] D. Godard, "Self-recovering equalization and carrier tracking in twodimensional data communication systems," IEEE Trans. Commun., vol. COM-28, pp. 1867-1875, Nov. 1980.

[32] J. R. Treichler and B. G. Agee, "A new approach to multipath correction of constant modulus signals," IEEE Trans. Acoustics, Speech and Signal Processing, vol. ASSP-31, pp. 459-472, Apr. 1983. 